\title{
Development of a Low-Cost GSM SMS-Based Humidity Remote Monitoring and Control system for Industrial Applications
}

\author{
Dr.B.Ramamurthy ${ }^{1}$ \\ Associate Professor \\ Dept of Instrumentation, S K University \\ Anantapur, Andhra Pradesh India \\ ramamurthy_ugc@yahoo.com
}

\author{
S.Bhargavi ${ }^{2}$ \\ Assistant Professor \\ Dept of E\&C, SJCIT \\ Chikballapur, Karnataka, India Chikballapur, Karnataka, India \\ bhargavisunil@gmail.com rshashiku@gmail.com
}

\begin{abstract}
The paper proposes a wireless solution, based on GSM (Global System for Mobile Communication) networks [1] for the monitoring and control of humidity in industries. This system provides ideal solution for monitoring critical plant on unmanned sites. The system is Wireless [2] therefore more adaptable and cost-effective. Utilizing Humidity sensor HSM-20G, ARM Controller LPC2148 and GSM technology this system offers a cost effective solution to wide range of remote monitoring and control applications. Historical and real time data can be accessed world wide using the GSM network. The system can also be configured to transmit data on alarm or at preset intervals to a mobile phone using SMS text messaging. The proposed system monitors and controls the humidity from the remote location and whenever it crosses the set limit the LPC2148 processor will sends an SMS to a concerned plant authority(s) mobile phone via GSM network. The concerned authority can control the system through his mobile phone by sending AT Commands to GSM MODEM and in turn to processor. Also the system provides password security against operator misuse/abuse. The system uses GSM technology [3] thus providing ubiquitous access to the system for security and automated monitoring and control of Humidity.
\end{abstract}

Keywords-Automation, GSM, SMS, Humidity Sensor (HSM-20G), ARM Controller LPC2148, Remote Monitoring \& Control, AT Commands, Password Security, Mobile phone.

\section{INTRODUCTION}

Remote Monitoring, Control and intelligent maintenance is one of the most important criteria for maximizing production and process plant availability. Wireless media has been undergoing a rapid innovation process in search for a reliable, simple and business-viable technology for fast, easy and inexpensive diagnosis of faults in industries.

Today, growth is coming from global expansion and services. A new surge of growth will come through new technology (Wireless) [4], production at the lowest cost for global distribution, and fast time-to-market. A Wireless Industrial Automation communications segment, at the present time, presents a mixture of standardized and proprietary technologies.

Recently, there has been much interest in remote monitoring and control in the field of the Industrial automation. There has also been much interest in wireless communication [5] in industrial sector for uses in automation as well as to increase the safety and security standards. There is a great deal of benefits for industries to adopt the wireless communication to control systems. Currently the common conditions of use of SCADA systems [6] only allow for control and supervision to take place when the operator and the plant being observed are in the same general vicinity. It led to the emergence of the wireless remote monitoring and control systems. This contribution develops the systematic design methods for the development of a low cost GSM SMS-based Humidity Remote Monitoring and Control system for industrial applications over the wireless communication. Measurement and control of relative humidity [7] has significant appliance in industry, science, healthcare, agriculture and controlling technological processes. This is the main objective and focus of the present work.

The use of mobile phones or handsets has grown exponentially over the years [8]. As the number of mobile phone users increased, the technology and infrastructure supporting the handsets have also evolved to cope with the traffic created by the number of users. On top of that, the demands of mobile phone users have also changed, and the average mobile phone of today can do many things that would have never been dreamt of 10 years ago.

The dominant mobile phone network in the world today is GSM. It is a digital mobile communication network, which developed, rapidly in recent years. This network has coverage in most urban areas and offer support for the SMS [9] that allows users to communicate with each other by sending short text messages to each other at minimal cost. The maximum length of the 
messages cannot exceed 150 alphanumeric Latin characters. This is enough to send short alert messages or commands to remote system. In the world of automation and control, several methods have thus far been employed to attain remote monitoring and control of various processes. These attempts have met with varying levels of success.

The primary aim of this paper is to propose the concept of Development of a Low-Cost GSM SMS-Based Humidity Remote Monitoring and Control system for Industrial Applications using the combination of a Embedded ARM Controller (ARM7 TDMI-S LPC2148) [10] and a GSM communications module linked by a serial communications port. Using this relative humidity could be efficiently recorded from the remote location and whenever it crosses the set limit, the ARM processor will send an SMS alert to a concerned authority(s) mobile phone. The concerned authority(s) can control the system through the mobile phone by sensing AT commands to the GSM MODEM. Also the system provides password security against operator misuse/abuse

The benefits of this paper are:

- Flexibility / modularity in control by the use of an ARM processor.

- Global coverage through the use of the GSM network.

- Extremely low cost device adapted for different applications.

- Scalable, Robust and Reliable.

- Provides password security.

- Efficient and cheap means of communication by use of SMS.

- True mobility using mobile phone sets.

- Ideal for monitoring and control critical plant on unmanned sites.

\section{FUNCTIONAL BLOCK DIAGRAM AND DESCRIPTION}

The Functional Block diagram of the entire system is as shown in the Figure 1. All the major subsystem blocks are shown with their interconnections to each module. The block diagram consists of Humidity Sensor (HSM-20G), ARM TDMI-S LPC2148 Processor, GSM MODEM (SIM300), MAX232 Level converter and inverter, Controlling device, Mobile phone, Line driver ULN2003 and Relay set and Personal computer. In this application, the system was set up to monitor and control the relative humidity and ensures that it was within safe operating limits. The detailed descriptions of the blocks used in the system are explained below.

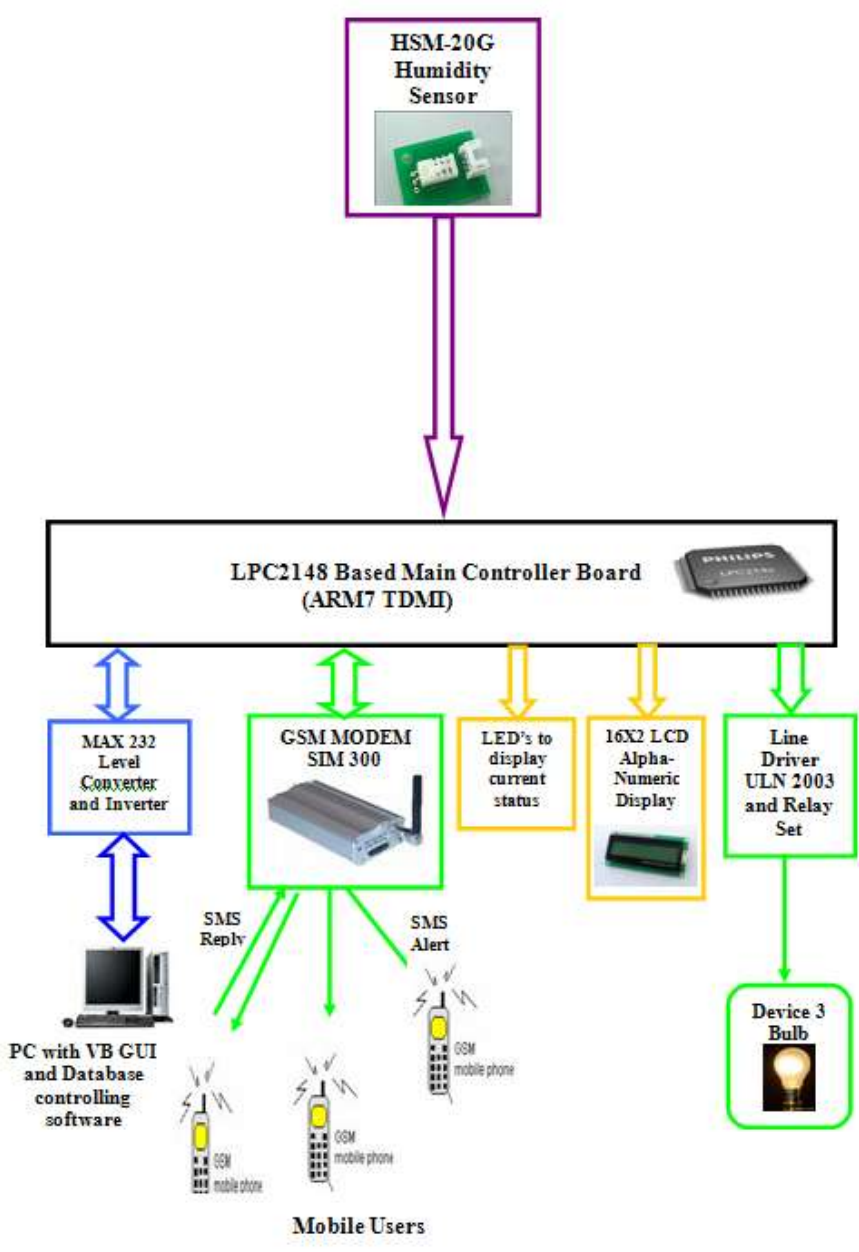

Figure 1: The Functional Block diagram

\section{A. ARM7 TDMI LPC2148 Processor}

The NXP (founded by Philips) LPC2148 is an ARM7TDMI-S based high-performance 32-bit RISC Microcontroller with Thumb extensions 512KB on-chip Flash ROM with In-System Programming (ISP) and In-Application Programming (IAP), 32KB RAM, Vectored Interrupt Controller, Two 10bit ADCs with 14 channels, USB 2.0 Full Speed Device Controller, Two UARTs, one with full modem interface. Two I2C serial interfaces, Two SPI serial interfaces Two 32-bit timers, Watchdog Timer, PWM unit, Real Time Clock with optional battery backup, Brown out detect circuit General purpose I/O pins. CPU clock up to $60 \mathrm{MHz}$, On-chip crystal oscillator and On-chip PLL. Due to their tiny size and low power consumption, LPC2148 are ideal for applications where miniaturization is a key requirement, such as access control and point-of-sale. Serial communications interfaces ranging from a USB 2.0 Full-speed device, multiple UARTs, SPI, SSP to I2C-bus and on-chip SRAM of $8 \mathrm{kB}$ up to $40 \mathrm{kB}$, make these devices very well suited for communication gateways and protocol converters, soft modems, voice recognition and low end imaging, providing both large buffer size and high processing power. Various 32-bit timers, single or dual 10-bit ADC(s), 10-bit 
DAC, PWM channels and 45 fast GPIO lines with up to nine edge or level sensitive external interrupt pins make these microcontrollers suitable for industrial control and medical systems.

\section{B. Sensors}

Sensors are used for process monitoring and for process control. These are essential elements of safe and profitable plant operation that can be achieved only if the proper sensors are selected and installed in the correct locations. In this paper the sensor is for the measurement of relative humidity.

\section{1) Humidity Sensor (HSM-20G)}

Humidity sensor [11] is a device consisting of a special plastic material whose electrical characteristics change according to the amount of humidity in the air. Basically it is a sensor that senses the amount of water vapor in air. The module of HSM-20G is essential for those applications where the relative humidity can be converted to standard voltage output. The humidity sensor module HSM-20G is shown in Figure 2.

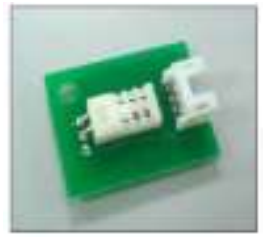

Figure 2: Humidity Sensor Module HSM-20G

The features of HSM-20G include:

$>$ Voltage analog output for both humidity and temperature.

$>$ Small size makes it easy to conceal

$>$ Compatible with all types of microcontrollers

$>$ High sensitivity to humidity in the air

\section{GSM MODEM}

A GSM modem is a specialized type of modem, which accepts a SIM card, and operates over a subscription to a mobile operator, just like a mobile phone. From the mobile operator perspective, a GSM modem looks just like a mobile phone. A GSM modem can be a dedicated modem device with a serial, USB or Bluetooth connection, or it may be a mobile phone that provides GSM modem capabilities. A GSM modem could also be a standard GSM mobile phone with the appropriate cable and software driver to connect to a serial port or USB port on computer. Any phone that supports the "extended AT command set" for sending/receiving SMS messages, as defined in the ETSI GSM 07.05 Specification can be supported by the Now SMS/MMS Gateway. In the proposed system we have used SIMCOM SIM300 GSM module.

SIM300 [12] is a Tri-band GSM/GPRS engine that works on frequencies EGSM $900 \mathrm{MHz}$, DCS $1800 \mathrm{MHz}$ and PCS1900 MHz. SIM300 provides GPRS multi-slot class 10 capability and support the GPRS coding schemes CS-1, CS-2, CS-3 and CS-4. With a tiny configuration of $40 \mathrm{~mm} \times 33 \mathrm{~mm} \times 2.85 \mathrm{~mm}$, SIM300 can fit almost all the space requirement in your application, such as Smart phone, PDA phone and other mobile device. The physical interface to the mobile application is made through a 60 pins board-to-board connector, which provides all hardware interfaces between the module and customers' boards. The SIM300 is designed with power saving technique, the current consumption to as low as $2.5 \mathrm{~mA}$ in SLEEP mode. The SIM300 is integrated with the TCP/IP protocol, Extended TCP/IP AT commands are developed for customers to use the TCP/IP protocol easily, which is very useful for those data transfer applications.

\section{Mobile Phone}

A mobile phone also known as a wireless phone, cell phone, or cellular telephone is a little portable radio telephone. Mobile Phone can serve as powerful tool for world-wide communication. The Mobile Phone is a natural choice, since it is a communication resource generally available by people, which makes them practically always contactable and capable to send commands to operate the parameters in the industries.

The use of mobile phones or handsets has grown exponentially over the years. As the number of mobile phone users increased, the technology and infrastructure supporting the handsets have also evolved to cope with the traffic created by the number of users. On top of that, the demands of mobile phone users have also changed, and the average mobile phone today can do many things that would have never been dreamt of 10 years ago.

The mobile phone can be used to communicate over long distances without wires. It works by communicating with a nearby base station (sometimes called a "cell") which connects it to the main phone network. As the mobile phone moves around, if the mobile phone gets too far away from the cell it is connected to, that cell sends a message to another cell to tell the new cell to take over the call. This is called a "hand off," and the call continues with the new cell the phone is connected to. The hand-off is done so well and carefully that the user will usually never even know that the call was transferred to another cell. Since a cell phone allows you to be anywhere, and to move around while calling, they became very popular.

\section{E. MAX 232 Level Converters}

Since the RS232 is not compatible with today's Microprocessors and Microcontrollers, we need a line driver or voltage converter to convert RS232's signals to TTL voltage levels. One example of such a converter is MAX 232 from Maxim corp. The MAX232 converter converts from RS232 voltage levels to TTL voltage levels and vice versa. One advantage of the MAX232 chip is that it uses a $+5 \mathrm{v}$ power source, which is the same as the source voltage for the microcontroller. In other words, 
with a single $+5 \mathrm{v}$ power supply we can power both the microcontroller and MAX232, with no need for the dual power supplies that are common in many older systems. The MAX 232 has two sets of line drivers for transferring and receiving data.

\section{F. Line Driver (ULN 2003)}

The ULN2003 is a monolithic high voltage and high current Darlington transistor arrays. It consists of seven NPN Darlington pairs that feature high-voltage outputs with common-cathode clamp diode for switching inductive loads. The collector-current rating of a single darlington pair is $500 \mathrm{~mA}$. The Darlington pairs may be paralleled for higher current capability. Applications include relay drivers, hammer drivers, lamp drivers, display drivers (LED gas discharge), line drivers, and logic buffers. The ULN2003 has a $2.7 \mathrm{kilo}$ ohms series base resistor for each Darlington pair for operation directly with TTL or $5 \mathrm{~V}$ CMOS devices. The features are $500 \mathrm{~mA}$ rated collector current (Single output), High-voltage outputs: 50V, Inputs compatible with various types of logic, Relay driver application.

\section{G. Controlling Device}

The device used to control the humidity in the industry is Alarm.

\section{H. Personal Computer}

A Personal computer is a programmable machine that receives input, stores and manipulates data, and provides output in a useful format. A personal computer may be a desktop computer, a laptop, a tablet PC, or a handheld PC. The most common microprocessors in personal computers are x86-compatible CPUs. Software applications for personal computers include word processing, spread sheets, data bases, Web browsers and e-mail clients, games, and myriad personal productivity and special-purpose software applications. Modern personal computers often have high-speed or dial-up connections to the Internet allowing access to the World Wide Web and a wide range of other resources. Personal computers may be connected to a local area network (LAN), either by a cable or a wireless connection.

The data logging is achieved continuously by the ARM7 TDMI LPC2148 processor to the personal Computer via the MAX232. This data is received by the software running on the PC and continuously updates a database by using Visual Basic and also we can generate reports and graphs automatically. Focusing on the client requirements, the following capabilities have been provided in the software.

- Monitoring - This is the main feature of the system where extracted information is presented for the operator in near real-time. Monitoring has been divided in two sections.
- Full graphical data representation - In this section, the user is able to monitor the plant in a very user friendly manner where details are represented in dynamic graphical interfaces in personal computer.

- Text base data representation - In this section, the near real time details are represented in tables without graphical objects.

\section{HARDWARE IMPLEMENTATION AND DESCRIPTION}

The circuit diagram of the entire system is as shown in the Figure 3.

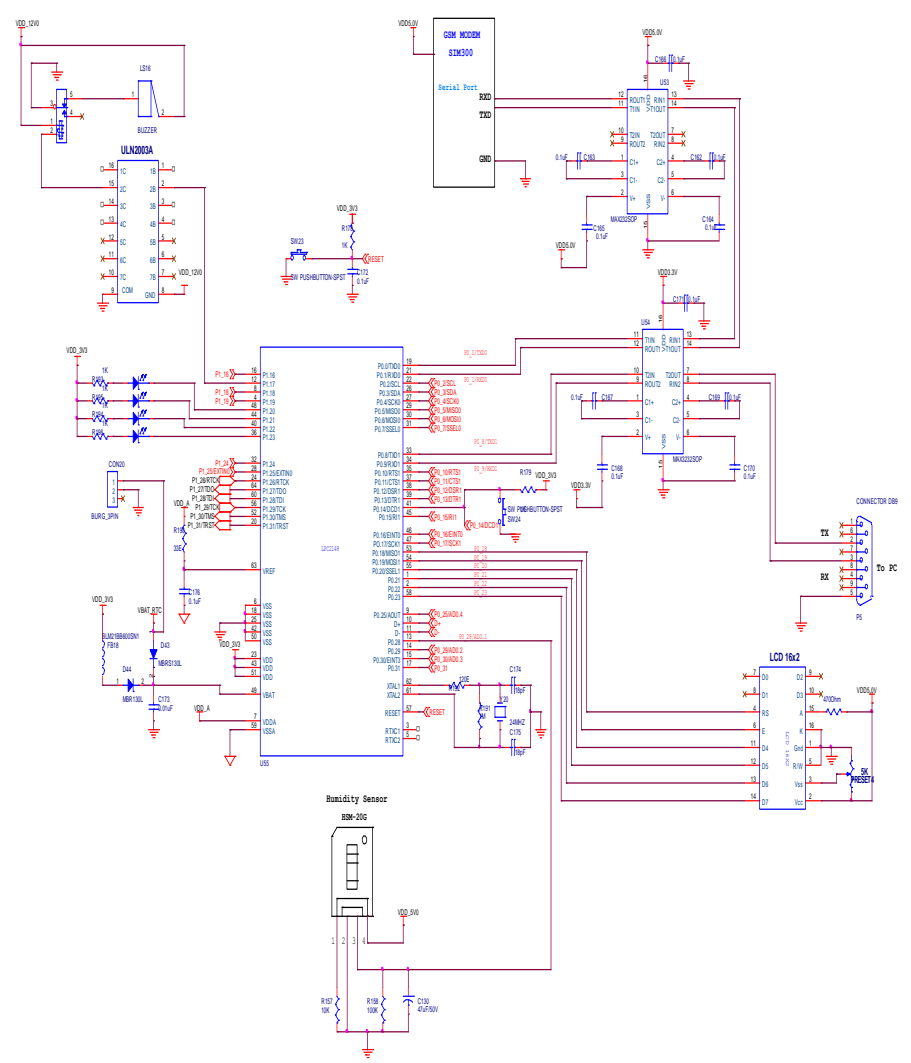

Figure 3: Circuit diagram of the entire system

The circuit diagram of the entire system is shown in Figure 3. Humidity is taken as a parameter and the humidity sensor HSM-20G will sense the humidity and will give a voltage output corresponding to the humidity value. This signal is taken into LPC2148 processor through the analog input channel for comparison. This signal is digitized using the inbuilt 10-bit ADC of the LPC2148 processor and compare the data with its threshold value for any status changes or value crossing the limit. If the value is more than the threshold limiting alert the concerned authority(s) by sending an SMS through GSM MODEM to his/her Mobile phone and switch the Bulb ON. If the values are within limits switch 
the Bulb OFF. The authority(s) concerned to the plant can control the threshold value by changing the humidity value or by switching ON the Bulb by sending AT commands to GSM MODEM, which will be directed to the processor. The authority(s) can also monitor the status of the humidity value remotely through his/her mobile phone by issuing a string of commands to GSM MODEM and in turn to the processor. The measured values are displayed in personal computer for further analysis to download reports and graphs.

\section{SOFTWARE DEVELOPMENT}

The software for the system is developed in Embedded $\mathrm{C}$ and Visual Basic. The flowcharts depicting the monitoring and the control of humidity are shown in Figure.4 and Figure.5.

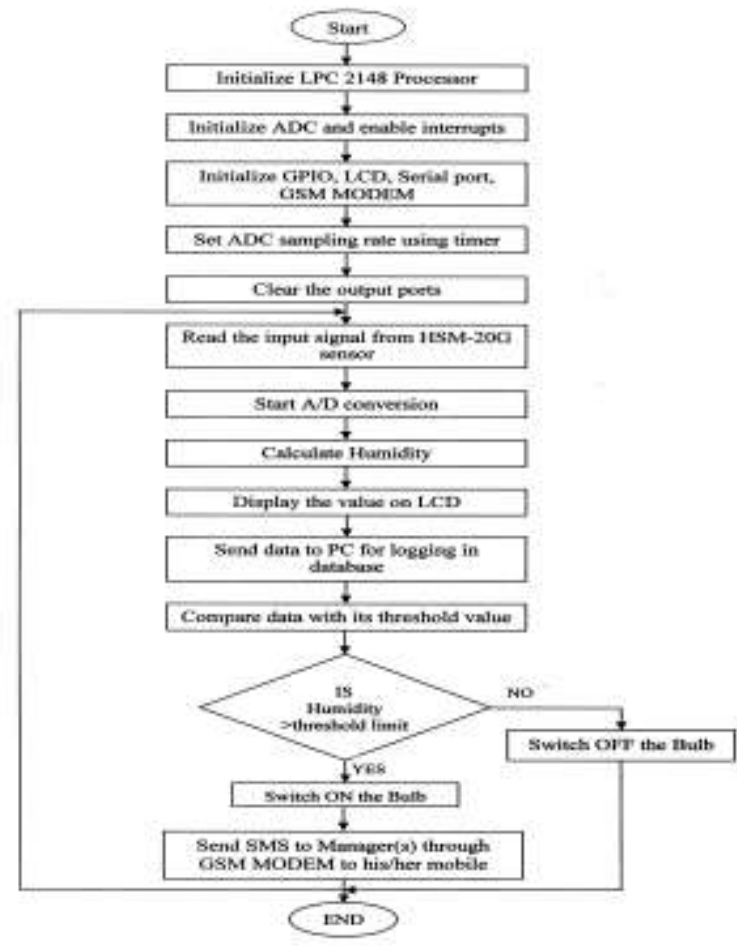

Figure 4: The flowchart for monitor process

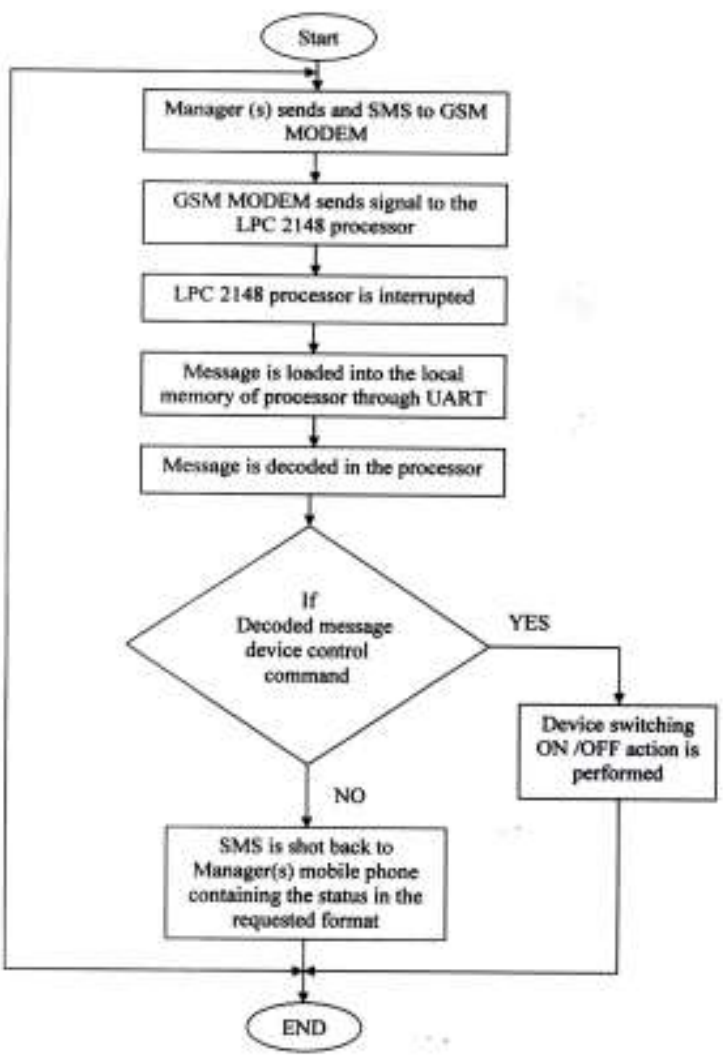

Figure 5: The flowchart for Control process

\section{RESULTS AND DISCUSSION}

The results obtained by using the proposed system are discussed in this section. Figure 6 shows the measurement and control of Humidity in the graphical representation, Figure 7 shows the status of the humidity in the mobile phone, Figure 8 shows the device status, current data and high limit values of sensors.

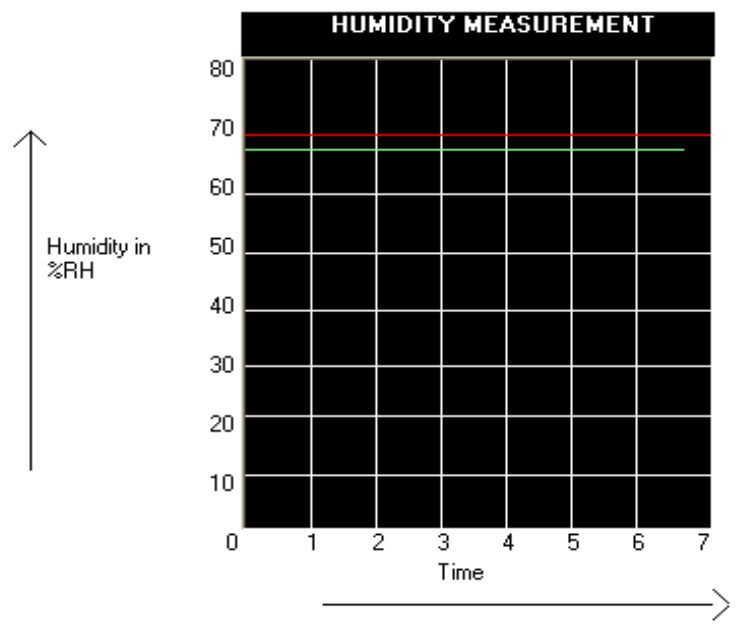

Figure 6: Graphical Representation of Humidity Measurement 


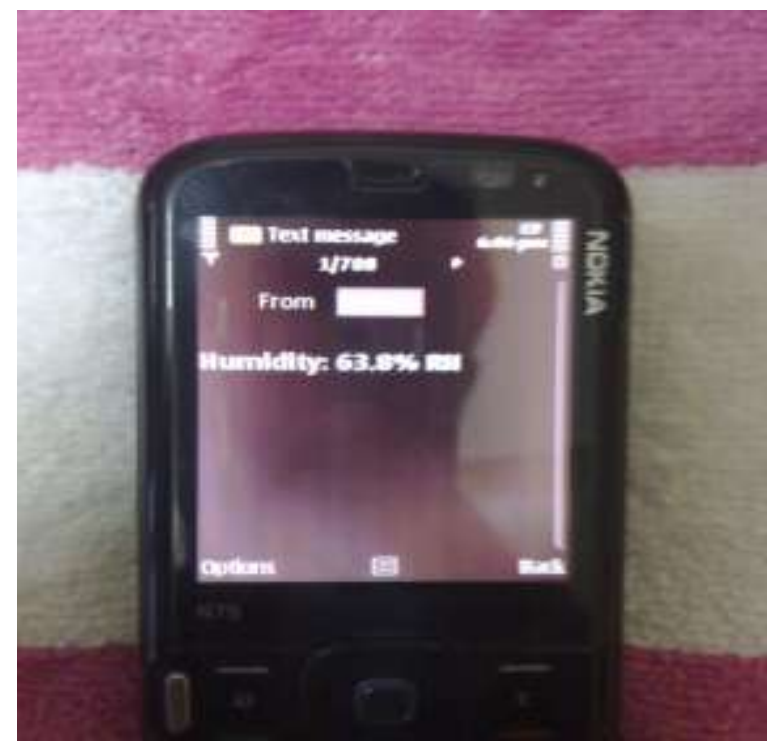

Figure 7: Status of Humidity in the Remote Mobile

Phone User

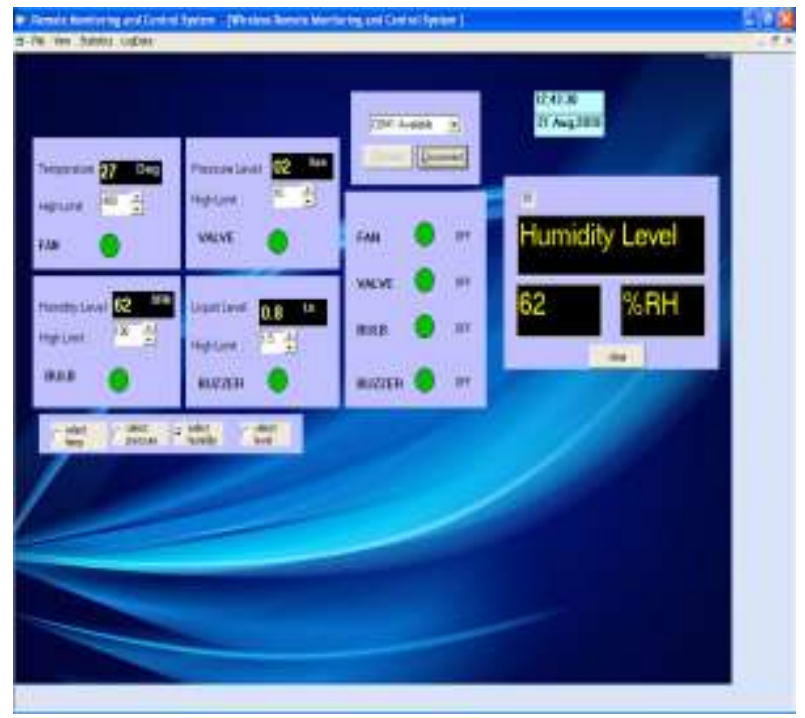

Figure 8: Device status, Current data and High limit values of sensors

In RMACS if there is any deviation observed in the measured value the remote user can change the set point value with his mobile phone by sending command " SET HUMI XXXX \%RH" where XXXX indicates the value. The designed RMACS tested with remote user mobile phone for different set points along with measured values of humidity with real time is shown in Figure 9. System is tested with the standard set point and also with different set point values in the present study. The results are tabulated in Table 1.

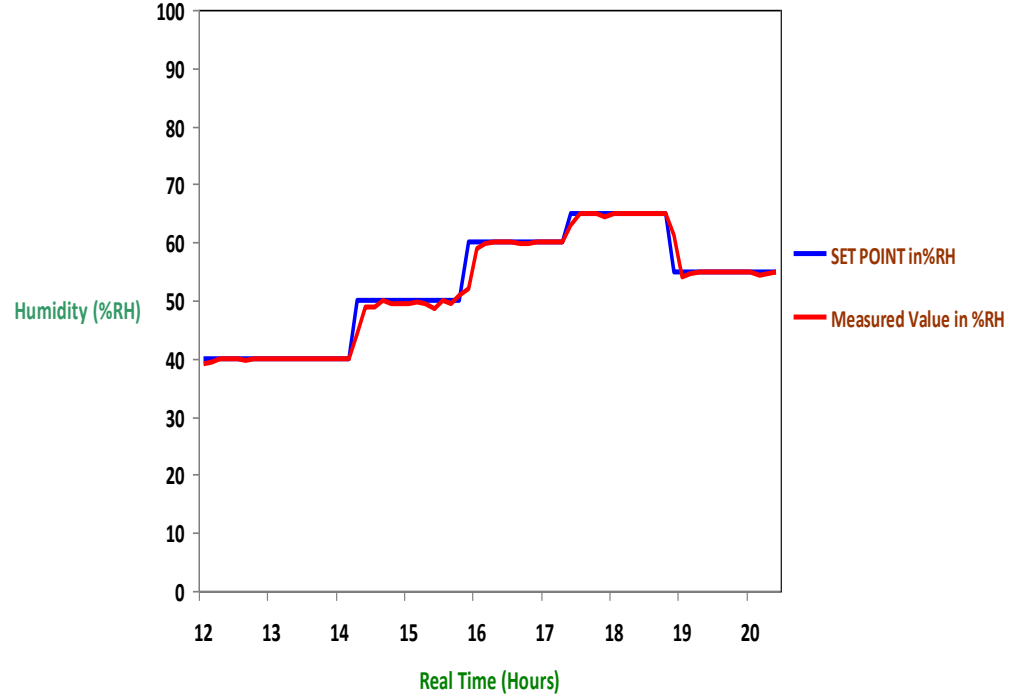

Figure 9:Graphical Representation of Humidity with different set point values

\begin{tabular}{|c|c|c|c|}
\hline Humidity \%RH & Bulb Status & SMS status & SMS Message \\
\hline 10 & OFF & NO & - \\
\hline 20 & OFF & NO & - \\
\hline 35 & OFF & N0 & - \\
\hline 40 & OFF & NO & - \\
\hline 65 & ON & YES & Humitity corsing limit \\
\hline 70 & ON & YES & Humidity crossed limit \\
\hline
\end{tabular}

Table 1: Results of the system

The above results confines that the monitoring and control device is always with the concerned authority(s) and also it is possible to read the data from any remote place. If the input value is near or more than the threshold limit then the processor will sends an SMS as "Humidity crossing limit" or "Humidity crossed limit" to a authority(s) mobile phone through GSM MODEM. The authority(s) concerned to the plant can control the set point by changing the input value or can switch $\mathrm{ON}$ the Bulb by sending AT commands to GSM MODEM, which will be directed to the processor. The authority(s) can also monitor the status of the Humidity remotely by issuing a string of commands to GSM modem and in turn to the processor. The measured values are stored in personal computer for further analysis to download the reports and graphs. The system was tested by measuring humidity up to $\% 90 \mathrm{RH}$ and the results are in good agreement with experimental values. 


\section{CONCLUSION}

The system has provided a low cost, secure, ubiquitously accessible, remotely monitored and controlled solution for automation of industries has been introduced. The use of a ARM Processor, GSM module, Sensors and actuators provide exciting possibilities. However as far as the industrial applications are concerned this can be viewed as a low cost, customized wireless RMACS system. Thus this solution can be customized to suit any other industrial requirement related to monitoring and controlling provided industrial sensors are in use.

The approach discussed in the paper is novel and has achieved the target to control humidity remotely using the GSM SMS-based system satisfying user needs and requirements. GSM technology capable solution has proved to be controlled remotely, provide security and is cost-effective as compared to the previously existing systems. Hence we can conclude that the required goals and objectives of the system have been achieved.

\section{REFERENCES}

[1] Drumea A, Popescu Camelia, Svasta P, GSM Solutions for Low Cost Embedded Systems for Industrial Control, 28 $8^{\text {th }}$ Int. Spring Seminar on Electronics technology, IEEE, 2005, pp. 226-230.

[2] Malik Sikandar Hayat Khiyal, Aihab Khan, Erum Shehzadi "SMS Based Wireless Home Appliance Control System (HACS) for Automating Appliances and Security", Issues in Informing Science and Information Technology, Volume 6, 2009.

[3] Chen Peijiang Xuehua, "Design and Implementation of Remote Monitoring System Based on GSM", 2008 IEEE Pacific-Asia Workshop on Computational Intelligence and Industrial Application

[4] Ciubotaru-Petrescu B, Chiciudean D, Cioarga R,Stanescu D,"Wireless Solutions for Telemetry in Civil Equipment and Infrastructure Monitoring", 3rd Romanian-Hungarian Joint Symposium on Applied Computational Intelligence (SACI) May 25-26, 2006.

[5] Catalin Pancu, Adrian Baraboi, Maricel Adam, Adrian Plesca, GSM Based Solution for Monitoring and Diagnostic of Electrical Equipment, Proceedings of the $13^{\text {th }}$ WSEAS International Conference on CIRCUITS.

[6] Daniel J.S.Lim, Vishy Karri,"Remote monitoring and control of hydrogen Safety Via SMS", $1^{\text {st }}$ International Conference on Sensing Technology November 21-23, 2005 Palmerston North, new Zealand.

[7] Grisha Spasov, Nilolay Kakanakov, Measurement of Temperature and Humidity using SHT 11/71 intelligent Sensor, ELECTRONICS'2004, 22-24 September, Sozopol, BULGARIA.

[8] Ken Wieland, "Spreading the Word"

Telecommunications Magazine International Edition October 2004, Issue Highlights.

[9] Puneet Gupta, SMS: How, what and where, Wireless Developer Network

[10] Trever Martin, The Insider's Guide to the Philips ARM7 Based Microcontroller, CEng MIEE, Feb 2005

[11]HSM-20G Humidity Sensor Module, datasheet from seeedstudio.com.
[12] http://www.shoky.com.tw/en/PDF/SIM300.pdf

AUTHORS PROFILE

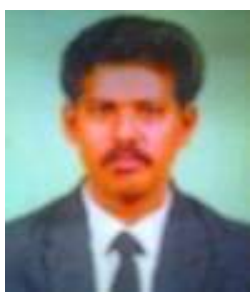

Dr.B.RamaMurthy is presently working as an Associative Professor in Department of Instrumentation \& USIC, SKU, Anantapur, Andhra Pradesh, India. $\mathrm{He}$ is having 18 years of Research \& teaching experience and 4 years of Industry experience. He guided 5 Ph.D.'s \& 4 M.Phil's. His areas of interest are Embedded Systems, Mobile Communication, Smart Cards and Industrial Instrumentation.

Prof.S.Bhargavi is presently

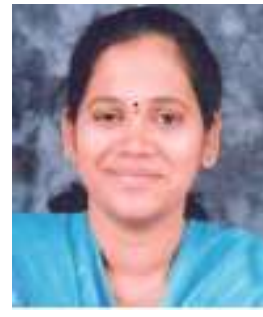
working as a Assistant Professor in the department of Electronics and Communication engg, SJCIT, Chikballapur, Karnataka, India. She is doing $\mathrm{PhD}$ in $\mathrm{SK}$ University, Anantapur. She is having 12 years of teaching experience. Her areas of interest are Embedded systems, Low Power VLSI, Wireless communication, ASIC and Cryptography.

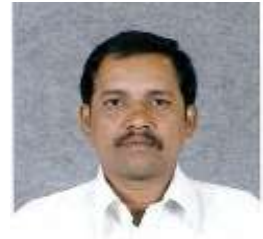

Dr.R.Shashikumar is presently working as a Professor in E \& C dept, SJCIT, Chikballapur, Karnataka, India. $\mathrm{He}$ is having 10 years of teaching and 6 years of Industry experience. His areas of interest includes ASIC, FPGA, Network Security. 